International Journal of Pure and Applied Mathematics

Volume 83 No. 2 2013, 303-306

ISSN: 1311-8080 (printed version); ISSN: 1314-3395 (on-line version)

url: http://www.ijpam.eu

doi: http://dx.doi.org/10.12732/ijpam.v83i2.10

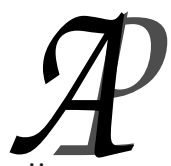

ijpam.eu

\title{
A COMPARISON OF COMPRESSED SENSING AND OTHER THEORIES
}

\author{
Hongying Xiao ${ }^{1}$, Shiwei Xie ${ }^{2} \S$ \\ ${ }^{1}$ College of Science \\ China Three Gorges University \\ Yichang, 443002, P.R. CHINA
}

\begin{abstract}
In this paper, we compare the methods of compressed sensing with other well-known results. Firstly,we review some classical theorems of linear algebra which deals with the existence and computation of solutions of linear equations. Afterwards, we will show what can compressed sensing do.
\end{abstract}

AMS Subject Classification: 15A06

Key Words: compressed sensing, sparse representation, linear equations, solution

\section{The Solvability of Linear Equations}

Linear equations plays a crucial role in pure mathematics and in applications as engineering, social science, etc. In this paper, we focus on two key problems: one is the solvability of linear equations, the other is the computation of such solutions. We firstly review a classical theorem in linear algebra, [5].

Theorem 1. Let $A$ be an $m \times n$ matrix, the equations

1. $A x=b$ is solvable $\Leftrightarrow R(A)=R(A, b)$;

2. $A x=b$ has a unique solution $\Leftrightarrow R(A)=R(A, b)=n$;

3. $A x=b$ has infinite solutions $\Leftrightarrow R(A)=R(A, b)<n$.

Received: October 19, 2012

(c) 2013 Academic Publications, Ltd. url: www.acadpubl.eu

${ }^{\S}$ Correspondence author 
By the above theorem, we investigate the existence of possible solutions of given linear equations. On the other hand, the next corollary presents a special class of homogeneous linear equations.

Corollary 2. Let $A$ be an $m \times n$ matrix with $m<n$, then the equations $A x=0$ has infinite solutions.

Proof. By assumption, we have $R(A)<m \leq n$.

\section{Computation of the Solutions}

In this section, we focus on how to compute the solutions of linear equations. we will firstly recall some classical matrix decompositions,

1. LU decomposition: $\mathrm{B}=\mathrm{LU}$, with $\mathrm{L}$ being lower-triangular and $\mathrm{U}$ being upper-triangular;

2. QR decomposition, $\mathrm{B}=\mathrm{QR}$, with $\mathrm{Q}$ being unitary and $\mathrm{R}$ being uppertriangular;

3. singular value decomposition ( $\mathrm{SVD}$ ), $B=U \Lambda V$, with $\mathrm{U}, \mathrm{V}$ being unitary and $\Lambda$ being diagonal.

Thu, any equations can be simplified as (1) A is diagonal ; (2) A is an uppertriangular matrix; (3) A is a unitary matrix.

\section{Compressed Sensing}

The theory of compressing emerges quite recently, [1],[3],[4]. It aims to overcome some intrinsic shortcomings of the classical Shannon sampling theorem. We will introduce below the new sampling method.

Let $x \in R^{N}$ be the primal signal which is unclear to us. Nevertheless, we know $y \in R^{n}$ which is obtained from a measurement matrix, i.e, $y=\Phi x$. In this case, $n \ll N$, so we call this representation under-sampled or sparse. By [5], classical linear algebra declare that we cannot determine $\mathrm{x}$ from $\mathrm{y}$. The compressed sensing theory is successful in that it can be used to reconstruct $\mathrm{x}$ under the following assumptions. 


\subsection{Sparsity of the Primary Signal}

Definition 3. Let there be a basis $\Psi=\psi_{i}$ such that the primary signal can be decomposed as $x=\sum_{i=1}^{L} \alpha_{i} \psi_{i}$ such that most of the coefficients $\alpha_{i}$ are close to 0 , then we call $\mathrm{x}$ a sparse signal under basis $\Psi$ and say that $\alpha_{i}$ is sparse.

In particular, we call the $\mathrm{x}$ to be s-sparse if at most $\mathrm{s}$ of the coefficients are nonzero. Similarly, we can discuss the situations where $\Psi$ is a frame of a dictionary. In all there circumstances, the primary signal can be compressed.

\subsection{Incoherence of the Measurement Matrix and Bases}

To simplify the statement, assume that both the measurement matrix $\Phi$ and the basis $\Psi$ has orthonormal column vectors of $R^{n}$.

Definition 4. The coherence is $\mu(\Phi, \Psi):=\sqrt{n} \max \left\langle\phi_{i}, \psi_{j}\right\rangle$, the largest number of the coherence between the column vectors of $\Phi$ and $\Psi$.

When $\mu$ is small, it is quite much possible to accomplish the under-sampled sampling. Thus, a key problem here is how to construct the appropriate $\Phi$ and $\Psi$.

Theorem 5. Let $\Phi$ be any measurement matrix and $\Psi$ be any stochastic matrix, then the coherent is low, i.e., $\mu=\sqrt{2 \log n}$.

Unfortunately, the stochastic method is unapplicable for implications. How to construct deterministic matrix remains to be an open problem.

\subsection{Sparse Reconstruction}

When the above two assumptions hold, the reconstruction can be transformed to the minimization of a convex function. By using $x=\Psi \alpha$, we can rewrite the equations $\Phi x=y$ as $\Phi \Psi \alpha=y$. We need only to find the sparse solution $\hat{\alpha}$ of the new equations. In 1999, Chen, Donoho and Saunders presented the following results called basis pursuit. That is, they proved that $\hat{\alpha}$ can be obtained by solving the minimization problem:

$$
(P 1)\left\{\begin{array}{l}
\hat{\alpha}=\operatorname{argmin}\|u\|_{1} \\
\Phi \Psi \alpha=y
\end{array}\right.
$$

Theorem 6. Let the signal $x$ be s-sparse under the orthornormal basis $\Psi$, let $\Phi$ be any stochastic matrix, and let $n>C \mu^{2} s \log N$, then the solution of (P1) is exact. 
Different methods in convex analysis can be approached to obtain a minimizer of the above convex functions, [2], so we have accomplished the reconstruction.

\section{References}

[1] Richard G. Baraniuk, Compressive sensing, IEEE Signal Processing Magazine, 4, No. 24 (2007).

[2] Heinz H. Bauschke, Patrick L. Combettes, Convex Analysis and Monotone Operator Theory in Hilbert Space, Springer (2010).

[3] Emmanuel Cands, Justin Romberg, Quantitative robust uncertainty principles and optimally sparse decompositions, Foundations of Comput. Math.,2, No. 6 (2006).

[4] D. Donoho, Compressed sensing, IEEE Transactions on Infromation Theory, 52, No. 4 (2006).

[5] Lee W. Johnson, R. Dean Riess, Jimmy T. Arnold, Introduction to Linear Algebra, Prentice-Hall (2002). 